\title{
Prevalence and predictors of food insecurity among people living with and without HIV in the African Cohort Study
}

\author{
Cecilia C Onyenakie ${ }^{1,2}$, Raphael U Nnakwe ${ }^{1,2}$, Nicole Dear $^{2,3}$, Allahna Esber $^{2,3}$, \\ Emmanuel Bahemana ${ }^{2,4}$, Hannah Kibuuka ${ }^{5}$, Jonah Maswai ${ }^{2,6}$, John Owuoth ${ }^{7,8}$, \\ Trevor A Crowell ${ }^{2,3}$, Christina S Polyak ${ }^{2,3}$, Julie A Ake ${ }^{2}$ and Michael Iroezindu 1,2,* \\ on behalf of the AFRICOS Study Group \\ 'HJF Medical Research International, Abuja, Nigeria: ${ }^{2}$ U.S. Military HIV Research Program, Walter Reed Army Institute \\ of Research, Silver Spring, MD, USA: ${ }^{3}$ Henry M. Jackson Foundation for the Advancement of Military Medicine, Inc., \\ Bethesda, MD, USA: ${ }^{4} \mathrm{HJF}$ Medical Research International, Mbeya, Tanzania: ${ }^{5}$ Makerere University Walter Reed \\ Project, Kampala, Uganda: ${ }^{6} \mathrm{HJF}$ Medical Research International, Kericho, Kenya: ${ }^{7}$ U.S. Army Medical Research \\ Directorate - Africa, Kisumu, Kenya: ${ }^{8} \mathrm{HJF}$ Medical Research International, Kisumu, Kenya
}

Submitted 24 November 2020: Final revision received 10 August 2021: Accepted 18 August 2021: First published online 23 August 2021

\begin{abstract}
Objective: We determined the prevalence and identified predictors of food insecurity in four African countries.

Design: Cross-sectional analyses at study enrolment.

Setting: From January 2013 to March 2020, people living with HIV (PLWH) and without HIV were enrolled at twelve clinics in Kenya, Uganda, Tanzania and Nigeria.

Participants: Participants reporting not having enough food to eat over the past 12 months or receiving $<3$ meals/d were defined as food insecure. Robust Poisson regression models were used to estimate unadjusted and adjusted prevalence ratios (aPR) and 95\% CI for predictors of food insecurity among all participants and separately among PLWH.

Results: $1694 / 3496$ participants (48.5\%) reported food insecurity at enrolment, with no difference by HIV status. Food insecurity was more common among older participants $(50+v$. 18-24 years aPR $1 \cdot 35,95 \%$ CI $1 \cdot 15,1 \cdot 59)$. Having $2-5$ (aPR $1 \cdot 14,95 \%$ CI 1.01, 1.30) or $>5$ dependents (aPR 1.17, $95 \%$ CI 1.02, 1.35), and residing in Kisumu West, Kenya (aPR 1·63, $95 \%$ CI 1·42, 1·87) or Nigeria (aPR 1·20, $95 \%$ CI 1.01, 1.41) was associated with food insecurity. Residing in Tanzania (aPR 0.65, $95 \%$ CI $0.53,0 \cdot 80$ ) and increasing education (secondary/above education $v$. none/ some primary education aPR $0 \cdot 73,95 \%$ CI $0 \cdot 66,0 \cdot 81$ ) was protective against food insecurity. Antiretroviral therapy (ART)-experienced PLWH were more likely to be food secure irrespective of viral load.

Conclusion: Food insecurity was highly prevalent in our cohort though not significantly associated with HIV. Policies aimed at promoting education, elderly care, ART access in PLWH and financial independence could potentially improve food security in Africa.
\end{abstract}

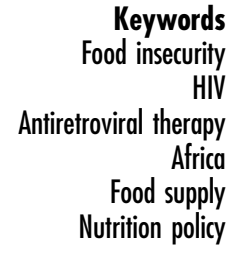

Food insecurity exists when an individual is unable to access or does not have enough money to buy an adequate amount of safe and nutritious food that meets their dietary needs and food preferences for an active and healthy life ${ }^{(1)}$. An estimated 821 million people around the globe were affected by food insecurity in 2017 , with a disproportionate burden in sub-Saharan Africa (SSA) ${ }^{(2,3)}$. The prevalence of severe food insecurity, specifically defined by global public health authorities as those who ran out of food and could not eat for a whole day or more in the past year, was only $1.4 \%$ in North America and Europe but $29.8 \%$ in Africa ${ }^{(2)}$. Except for North America and Europe, the burden of food insecurity has progressively increased in all other regions of the world between 2014 and $2017^{(2-5)}$. In SSA, the 
prevalence of severe food insecurity increased from $25.0 \%$ in 2014 to $33.8 \%$ in 2017 , compared to a much smaller increase from $11.2 \%$ to $12.4 \%$ in Northern Africa over the same period ${ }^{(2,3)}$. A $2018 \mathrm{UN}$ report revealed that 153 million people representing approximately $26 \%$ of those over 15 years in SSA were affected by severe food insecurity between 2014 and $2015^{(6)}$

Poverty, underdeveloped and underperforming agricultural sectors have been identified as underlying causes of food insecurity in developing countries, particularly those in $\mathrm{SSA}^{(7,8)}$. Agricultural output in SSA is greatly impacted by climate change, a high burden of HIV, social conflicts and poor governance ${ }^{(8)}$. Previous studies have also highlighted sociodemographic characteristics of households and health status as predictors of food insecurity ${ }^{(9-12)}$. In the USA, low income, unmet medical needs, poor health and limited participation in food assistance programs were associated with food insecurity ${ }^{(9)}$. In SSA, food insecurity has been more commonly observed in households with lower income, lower education, poor health status and larger family size/number of dependents ${ }^{(11,12)}$.

SSA carries a disproportionate burden of HIV infection, accounting for two-thirds of the global burden of the disease $^{(13)}$. A two-way relationship between food insecurity and HIV has been reported. On the one hand, HIV-related morbidity and mortality have been observed to negatively impact the socio-economic status of households and take a toll on the agricultural labour force, thereby predisposing households to food insecurity ${ }^{(11)}$. Among farming households in rural Nigeria, a decline in food production efficiency and utilised farm area, as well as in financial contributions to households were observed among people living with HIV (PLWH) ${ }^{(12)}$. On the other hand, food insecurity could potentially increase the risk of HIV transmis$\operatorname{sion}^{(13-15)}$, by triggering risk behaviours such as transactional sex ${ }^{(16,17)}$. Food insecurity has also been identified as a barrier to HIV care and may be associated with poor treatment outcomes ${ }^{(17-19)}$.

To date, studies that assessed food insecurity in SSA have tended to be small, limited to single countries and rarely involved a mixed population of people living with and without $\mathrm{HIV}^{(11,12,20-25)}$. Most studies that explored the relationship between HIV and food insecurity were either conducted in the era of limited access to antiretroviral therapy (ART) or had a preponderance of PLWH with advanced disease ${ }^{(14,16,19,26-28)}$.

A large, multinational evaluation of the prevalence and predictors of food insecurity could inform interventions to combat the growing epidemic of food insecurity in SSA. Moreover, in light of the ongoing COVID-19 pandemic, which has disrupted socio-economic activities and food security in SSA ${ }^{(29,30)}$, there is a critical need to identify at-risk groups for interventions to improve food security in the region. We determined the prevalence and identified predictors of food insecurity in people living with and without $\mathrm{HIV}$ in four African countries.

\section{Methods}

\section{Study design and participants}

The ongoing African Cohort Study (AFRICOS) is a longitudinal observational study that enrols PLWH and a smaller group of adults without HIV at twelve President's Emergency Plan for AIDS Relief-supported clinical care sites in Uganda, Kenya, Tanzania and Nigeria as previously described ${ }^{(31)}$. The vast majority of PLWH in our sample were invited to the study based on random selection from existing clinic lists (stratified by gender and ART status) or new enrolees to the clinic, while a minority (less than $5 \%$ ) are recruited from other HIV studies. Participants without HIV were recruited from individuals who screened negative for HIV at the counselling and testing units of the clinics. A few participants without HIV were serodiscordant partners of enrolled PLWH. All the President's Emergency Plan for AIDS Relief clinics where AFRICOS is conducted serve the general population. Recruitment/enrolment is ongoing for up to a maximum of 4200 participants (3500 PLWH and 700 people without HIV). Individuals were eligible if they were aged $\geq 18$ years and consented to data and specimen collection. An additional inclusion criterion for PLWH was the ongoing receipt of HIV care at the enrolling clinic. We excluded individuals who were pregnant at enrolment.

At enrolment and every 6 months thereafter, participants underwent medical history taking, physical examination and laboratory assessments, including HIV screening and confirmatory tests. Participants also completed a broad demographic and socio-behavioral questionnaire. Data from enrolment visits that occurred between 23 January 2013 and 1 March 2020 were included in these cross-sectional analyses. All participants provided written informed consent prior to enrolment.

\section{Data collection and definitions}

Demographic variables were obtained from participants by self-report, including age, sex, marital status, education, employment status, primary occupation, total number of people in the household and total number of dependents. For PLWH, clinical/laboratory data on WHO clinical staging, ART status, self-reported ART adherence, CD 4 count and viral load (VL) were also collected. Although information on the presence of opportunistic infection was obtained in PLWH, it was not reported as a separate variable since it is a component of WHO clinical staging. PLWH were considered suppressed if they had a VL $<1000$ copies/ml.

The following distinct questions were initially used to assess three food insecurity metrics: (i) 'Have you had enough food to eat over the past 12 months?' (ii)'On average, how many meals do you have in a day?' and (iii) 'Of 
these meals, how many have been cut or reduced in size because there is not enough food or money for food?' Possible responses to the first question were either 'yes' or 'no', and were coded as binary in the models. Responses to the last two questions were collected as a discrete number of meals. For analyses, the number of meals per day variable was categorised as $<3,3$ or $>3$, while the number of meals cut or reduced in size per day variable was categorised as none or $\geq 1$. Using a combined index, food insecurity was defined as a report of not having enough food to eat over the past 12 months or having less than three meals per day on average, while food security was defined as a report of having enough food to eat over the past 12 months and having three or more meals per day on average. The metric assessing the number of meals reduced in size per day because there is not enough food or money for food was excluded from the combined food insecurity index due to the disproportionately low number of participants with available data for this variable, as this question was added to the subject questionnaire in late 2017.

\section{Statistical analyses}

Descriptive statistics ( $\chi^{2}$ tests for categorical variables and Wilcoxon rank-sum tests for continuous variables) were used to describe differences between participants classified as food insecure using the combined food insecurity index and those classified as food secure. Generalised linear models with a Poisson distribution and robust error variances were used to estimate unadjusted and adjusted prevalence ratios (aPR) and 95\% confidence interval (CI) for associations between prespecified factors of interest and not having enough food to eat in the past 12 months and the combined food insecurity index. The PR is a measure of association quantifying the relationship between a predictor variable and a dichotomous outcome of interest in a cross-sectional analysis, especially suitable for outcomes with a high prevalence. Poisson regression models for count outcomes were used to estimate unadjusted and adjusted rate ratios for associations between pre-specified factors and the number of meals per day.

Independent variables were selected for inclusion in the model based on a review of existing literature, including HIV status, age, sex, marital status, education, employment status, primary occupation, farming status, household size and number of dependents. To potentially account for country-specific and seasonal/climatic variabilities, we also adjusted for site and year of enrolment. To evaluate HIV-specific factors, a subgroup analysis was performed among PLWH. A $p$-value $<0.05$ was considered statistically significant. All analyses were performed using SAS 9.3 (SAS, Cary, NC) and Stata $15 \cdot 0$ (StataCorp, College Station, TX).

\section{Results}

\section{Characteristics of the study population}

As of 1 March 2020, a total of 3551 participants were enrolled in the study, including 2937 PLWH and 614 participants without HIV. Of these, 3496 participants comprising 2884 PLWH and 612 people without HIV had complete data for analyses. Among all participants, the median age was 37.8 years (interquartile range $30.5-45.8$ years). The majority of participants were female ( $n$ 2029, 58.0\%) and $2070(59.2 \%)$ were married. A total of 1390 (39.8\%) reported being employed at the time of enrolment, including $356(10 \cdot 2 \%)$ who reported that their current primary occupation was farming. The median household size was 5 (interquartile range 3-6), and the median number of dependents was 2 (interquartile range 2-3) (Table 1 ).

The median age among PLWH was 38.3 years (interquartile range 31.1-46.1 years). Among PLWH, 889 (30.8\%) were not on ART at enrolment, while 1691 (58.6\%) were on ART with a VL $<1000$ copies/ml and 304 (10.5\%) were on ART with a VL $\geq 1000$ copies/ml. Only 564 (19.6\%) had a CD4 count below 200 cells $/ \mathrm{mm}^{3}$. WHO clinical stages III and IV disease were documented in 998 (34.6\%) and 183 (6.3\%) PLWH, respectively (Table 2).

\section{Prevalence of food insecurity}

Of 3496 participants, 1204 (34.4\%) reported not having enough food to eat in the past 12 months, while receiving $<3$ meals/d on average was documented in 1004 (28.7\%) participants. Reduction in number or size of $\geq 1$ meals because there was not enough food or money for food was observed in 68 (22.8\%) out of 298 participants with available data.

Statistically significant inter-site differences were observed in the proportion of individuals who reported not having enough food to eat over 12 months, with a greater proportion of participants in Kisumu West, Kenya ( $n$ $473,75.3 \%)$ and South Rift Valley, Kenya ( $n$ 405, 33.1\%) affected than in other sites $(P<0.001$, Fig. 1A). Similarly, the average number of meals received per day varied significantly by site, with a greater proportion of participants in Uganda ( $n 300,47 \cdot 0 \%)$ and Nigeria ( $n 145,39 \cdot 1 \%$ ) reporting $<3$ meals/d on average as compared to the other sites $(P<0.001$, Fig. 1B). There was no significant inter-site difference in the proportion of individuals who reported a cut or reduction in the size of meals ( $P=0 \cdot 18$, Fig. $1 \mathrm{C})$.

Based on the combined index, the prevalence of food insecurity was $48.5 \%$ ( $n$ 1694), with no statistically significant difference between participants with and without HIV ( $48.7 \% v .47 .4 \%, P=0.56$ ). A statistically significant difference was observed in the prevalence of food insecurity by site, with the highest prevalence in Kisumu West, Kenya ( $n$ 494, 78.7\%), followed by Uganda ( $n$ 332, 52.0\%) and the lowest prevalence in Tanzania ( $n$ 168, 26.4\%), $(P<0 \cdot 001$, Fig. 1D). 
Table 1 Characteristics of the study population by food insecurity status

\begin{tabular}{|c|c|c|c|c|c|c|c|}
\hline & \multicolumn{2}{|c|}{ All (n 3496) } & \multicolumn{2}{|c|}{$\begin{array}{c}\text { Food secure } \\
(n \text { 1802) }\end{array}$} & \multicolumn{2}{|c|}{$\begin{array}{l}\text { Food insecure } \\
(n \text { 1694) }\end{array}$} & \multirow[b]{2}{*}{$p$-value } \\
\hline & $n$ & $\%$ & $n$ & $\%$ & $n$ & $\%$ & \\
\hline HIV status & & & & & & & 0.56 \\
\hline People living with HIV & 2884 & 82.5 & 1480 & $82 \cdot 1$ & 1404 & 82.9 & \\
\hline People living without HIV & 612 & $17 \cdot 5$ & 322 & $17 \cdot 9$ & 290 & $17 \cdot 1$ & \\
\hline Age (years) & & & & & & & $<0.001$ \\
\hline $18-24$ & 400 & 11.4 & 268 & 14.9 & 132 & $7 \cdot 8$ & \\
\hline $25-39$ & 1641 & $46 \cdot 9$ & 856 & 47.5 & 785 & $46 \cdot 3$ & \\
\hline $40-49$ & 908 & $26 \cdot 0$ & 429 & $23 \cdot 8$ & 479 & $28 \cdot 3$ & \\
\hline $50+$ & 547 & $15 \cdot 6$ & 249 & $13 \cdot 8$ & 298 & $17 \cdot 6$ & \\
\hline Sex & & & & & & & 0.05 \\
\hline Male & 1467 & $42 \cdot 0$ & 728 & 40.4 & 739 & 43.6 & \\
\hline Female & 2029 & $58 \cdot 0$ & 1074 & $59 \cdot 6$ & 955 & $56 \cdot 4$ & \\
\hline Study site & & & & & & & $<0.001$ \\
\hline Kayunga, Uganda & 638 & $18 \cdot 2$ & 306 & $17 \cdot 0$ & 332 & $19 \cdot 6$ & \\
\hline South Rift Valley, Kenya & 1222 & $35 \cdot 0$ & 704 & $39 \cdot 1$ & 518 & 30.6 & \\
\hline Kisumu West, Kenya & 628 & $18 \cdot 0$ & 134 & 7.4 & 494 & $29 \cdot 2$ & \\
\hline Mbeya, Tanzania & 637 & $18 \cdot 2$ & 469 & $26 \cdot 0$ & 168 & 9.9 & \\
\hline Abuja and Lagos Nigeria & 371 & $10 \cdot 6$ & 189 & 10.5 & 182 & $10 \cdot 7$ & \\
\hline Year enrolled in AFRICOS & & & & & & & $<0.001$ \\
\hline 2013 & 310 & 8.9 & 148 & 8.2 & 162 & $9 \cdot 6$ & \\
\hline 2014 & 1037 & $29 \cdot 7$ & 521 & 28.9 & 516 & 30.5 & \\
\hline 2015 & 968 & $27 \cdot 7$ & 480 & $26 \cdot 6$ & 488 & $28 \cdot 8$ & \\
\hline 2016 & 692 & $19 \cdot 8$ & 335 & $18 \cdot 6$ & 357 & $21 \cdot 1$ & \\
\hline 2017 & 279 & $8 \cdot 0$ & 163 & $9 \cdot 0$ & 116 & $6 \cdot 8$ & \\
\hline 2018 & 97 & $2 \cdot 8$ & 70 & 3.9 & 27 & 1.6 & \\
\hline $2019 / 2020$ & 113 & 3.2 & 85 & 4.7 & 28 & 1.7 & \\
\hline Marital status & & & & & & & 0.01 \\
\hline Not married & 1426 & $40 \cdot 8$ & 771 & $42 \cdot 8$ & 655 & $38 \cdot 7$ & \\
\hline Married & 2070 & $59 \cdot 2$ & 1031 & $57 \cdot 2$ & 1039 & $61 \cdot 3$ & \\
\hline Education & & & & & & & $<0.001$ \\
\hline None or some primary & 1134 & 32.4 & 439 & 24.4 & 695 & 41.0 & \\
\hline Primary or some secondary & 1389 & $39 \cdot 7$ & 782 & 43.4 & 607 & 35.8 & \\
\hline Secondary and above & 973 & $27 \cdot 8$ & 581 & $32 \cdot 2$ & 392 & $23 \cdot 1$ & \\
\hline Employment status & & & & & & & 0.61 \\
\hline Not employed & 2106 & $60 \cdot 2$ & 1093 & $60 \cdot 7$ & 1013 & $59 \cdot 8$ & \\
\hline Employed & 1390 & 39.8 & 709 & $39 \cdot 3$ & 681 & $40 \cdot 2$ & \\
\hline Current primary occupation & & & & & & & $<0.001$ \\
\hline Unemployed & 2106 & $60 \cdot 2$ & 1093 & $60 \cdot 7$ & 1013 & $59 \cdot 8$ & \\
\hline Unskilled labour & 214 & $6 \cdot 1$ & 86 & 4.8 & 128 & $7 \cdot 6$ & \\
\hline Professional/managerial & 231 & $6 \cdot 6$ & 148 & 8.2 & 83 & 4.9 & \\
\hline Farmer & 356 & $10 \cdot 2$ & 156 & 8.7 & 200 & 11.8 & \\
\hline Commerce/business & 238 & $6 \cdot 8$ & 128 & $7 \cdot 1$ & 110 & $6 \cdot 5$ & \\
\hline Skilled trade & 178 & $5 \cdot 1$ & 92 & $5 \cdot 1$ & 86 & $5 \cdot 1$ & \\
\hline Other & 173 & 4.9 & 99 & $5 \cdot 5$ & 74 & 4.4 & \\
\hline Currently a farmer & & & & & & & $<0.01$ \\
\hline No & 3140 & 89.8 & 1646 & 91.3 & 1494 & $88 \cdot 2$ & \\
\hline Yes & 356 & $10 \cdot 2$ & 156 & 8.7 & 200 & 11.8 & \\
\hline Total no. people in household & & & & & & & 0.02 \\
\hline$\leq 6$ & 2721 & $77 \cdot 8$ & 1431 & 79.4 & 1290 & $76 \cdot 2$ & \\
\hline$>6$ & 775 & $22 \cdot 2$ & 371 & $20 \cdot 6$ & 404 & $23 \cdot 8$ & \\
\hline No. of dependents & & & & & & & $<0.001$ \\
\hline$<2$ people & 460 & $13 \cdot 2$ & 292 & $16 \cdot 2$ & 168 & 9.9 & \\
\hline 2-5 people & 1868 & 53.4 & 966 & $53 \cdot 6$ & 902 & $53 \cdot 2$ & \\
\hline$>5$ people & 1168 & 33.4 & 544 & $30 \cdot 2$ & 624 & $36 \cdot \overline{8}$ & \\
\hline
\end{tabular}

Data are presented as $\mathrm{n}$ (column \%). Pearson's $\chi^{2}$ and Wilcoxon rank-sum tests were used to describe differences between participants classified as food insecure and those classified as food secure, with food insecurity defined as a report of not having enough food to eat over the past 12 months or having less than three meals per day on average. Statistically significant $p$-values are presented in bold. AFRICOS, African Cohort Study.

Predictors of not having enough food over the past 12 months

Compared to participants 18-24 years old, not having enough food to eat in the past 12 months was more common among those 25-39 years old (aPR 1.35, 95\% CI 1.10, 1.64), 40-49 years old (aPR 1.58, $95 \%$ CI 1.28,
1.94) and 50+ years old (aPR 1.33, 95\% CI 1.07, 1.65; Table 3). As compared to having less than two dependents, having 2-5 dependents (aPR 1.27, $95 \%$ CI 1.06, 1.52) or more than five dependents (aPR 1.41, $95 \%$ CI 1.16, 1.71) was associated with not having enough food to eat in the past 12 months. Residing in South Rift Valley, Kenya 
Table 2 Characteristics of the study population living with HIV by food insecurity status

\begin{tabular}{|c|c|c|c|c|c|c|c|}
\hline & \multicolumn{2}{|c|}{ All $(n$ 2884) } & \multicolumn{2}{|c|}{$\begin{array}{c}\text { Food secure } \\
(n 1480)\end{array}$} & \multicolumn{2}{|c|}{$\begin{array}{l}\text { Food insecure } \\
\quad(n 1404)\end{array}$} & \multirow{2}{*}{$p$-value } \\
\hline & $n$ & $\%$ & $n$ & $\%$ & $n$ & $\%$ & \\
\hline Age (years) & & & & & & & $<0.001$ \\
\hline $18-24$ & 307 & $10 \cdot 6$ & 211 & $14 \cdot 3$ & 96 & $6 \cdot 8$ & \\
\hline $25-39$ & 1320 & $45 \cdot 8$ & 670 & $45 \cdot 3$ & 650 & $46 \cdot 3$ & \\
\hline $40-49$ & 790 & $27 \cdot 4$ & 382 & $25 \cdot 8$ & 408 & $29 \cdot 1$ & \\
\hline $50+$ & 467 & $16 \cdot 2$ & 217 & 14.7 & 250 & $17 \cdot 8$ & \\
\hline Sex & & & & & & & 0.37 \\
\hline Male & 1198 & 41.5 & 603 & 40.7 & 595 & $42 \cdot 4$ & \\
\hline Female & 1686 & $58 \cdot 5$ & 877 & $59 \cdot 3$ & 809 & $57 \cdot 6$ & \\
\hline Study site & & & & & & & $<0.001$ \\
\hline Kayunga, Uganda & 525 & $18 \cdot 2$ & 253 & $17 \cdot 1$ & 272 & 19.4 & \\
\hline South Rift Valley, Kenya & 1014 & $35 \cdot 2$ & 577 & $39 \cdot 0$ & 437 & $31 \cdot 1$ & \\
\hline Kisumu West, Kenya & 503 & 17.4 & 99 & 6.7 & 404 & $28 \cdot 8$ & \\
\hline Mbeya, Tanzania & 541 & $18 \cdot 8$ & 397 & $26 \cdot 8$ & 144 & $10 \cdot 3$ & \\
\hline Abuja and Lagos Nigeria & 301 & $10 \cdot 4$ & 154 & $10 \cdot 4$ & 147 & $10 \cdot 5$ & \\
\hline Year enrolled in AFRICOS & & & & & & & $<0.001$ \\
\hline 2013 & 266 & 9.2 & 123 & $8 \cdot 3$ & 143 & $10 \cdot 2$ & \\
\hline 2014 & 830 & $28 \cdot 8$ & 420 & 28.4 & 410 & $29 \cdot 2$ & \\
\hline 2015 & 803 & $27 \cdot 8$ & 406 & $27 \cdot 4$ & 397 & $28 \cdot 3$ & \\
\hline 2016 & 614 & $21 \cdot 3$ & 284 & $19 \cdot 2$ & 330 & 23.5 & \\
\hline 2017 & 216 & 7.5 & 133 & $9 \cdot 0$ & 83 & $5 \cdot 9$ & \\
\hline 2018 & 70 & $2 \cdot 4$ & 50 & 3.4 & 20 & 1.4 & \\
\hline $2019 / 2020$ & 85 & 2.9 & 64 & $4 \cdot 3$ & 21 & 1.5 & \\
\hline Marital status & & & & & & & 0.01 \\
\hline Not married & 1258 & 43.6 & 681 & $46 \cdot 0$ & 577 & $41 \cdot 1$ & \\
\hline Married & 1626 & $56 \cdot 4$ & 799 & $54 \cdot 0$ & 827 & 58.9 & \\
\hline Education & & & & & & & $<0.001$ \\
\hline None or some primary & 960 & $33 \cdot 3$ & 370 & $25 \cdot 0$ & 590 & $42 \cdot 0$ & \\
\hline Primary or some secondary & 1138 & 39.5 & 643 & 43.4 & 495 & $35 \cdot 3$ & \\
\hline Secondary and above & 786 & $27 \cdot 3$ & 467 & $31 \cdot 6$ & 319 & $22 \cdot 7$ & \\
\hline Employment status & & & & & & & 0.56 \\
\hline Not employed & 1741 & $60 \cdot 4$ & 901 & 60.9 & 840 & 59.8 & \\
\hline Employed & 1143 & 39.6 & 579 & $39 \cdot 1$ & 564 & $40 \cdot 2$ & \\
\hline Current primary occupation & & & & & & & $<0.001$ \\
\hline Unemployed & 1741 & $60 \cdot 4$ & 901 & 60.9 & 840 & $59 \cdot 8$ & \\
\hline Unskilled labour & 185 & $6 \cdot 4$ & 74 & $5 \cdot 0$ & 111 & 7.9 & \\
\hline Professional/managerial & 189 & $6 \cdot 6$ & 120 & $8 \cdot 1$ & 69 & 4.9 & \\
\hline Farmer & 272 & $9 \cdot 4$ & 114 & $7 \cdot 7$ & 158 & $11 \cdot 3$ & \\
\hline Commerce/business & 205 & $7 \cdot 1$ & 109 & $7 \cdot 4$ & 96 & $6 \cdot 8$ & \\
\hline Skilled trade & 151 & $5 \cdot 2$ & 80 & $5 \cdot 4$ & 71 & $5 \cdot 1$ & \\
\hline Other & 141 & 4.9 & 82 & 5.5 & 59 & $4 \cdot 2$ & \\
\hline Currently a farmer & & & & & & & $<0.01$ \\
\hline No & 2612 & $90 \cdot 6$ & 1366 & $92 \cdot 3$ & 1246 & $88 \cdot 7$ & \\
\hline Yes & 272 & 9.4 & 114 & $7 \cdot 7$ & 158 & $11 \cdot 3$ & \\
\hline Total no. people in household & & & & & & & 0.06 \\
\hline$\leq 6$ & 2281 & $79 \cdot 1$ & 1191 & $80 \cdot 5$ & 1090 & $77 \cdot 6$ & \\
\hline$>6$ & 603 & $20 \cdot 9$ & 289 & 19.5 & 314 & $22 \cdot 4$ & \\
\hline No. of dependents & & & & & & & $<0.001$ \\
\hline$<2$ people & 380 & $13 \cdot 2$ & 245 & $16 \cdot 6$ & 135 & $9 \cdot 6$ & \\
\hline $2-5$ people & 1580 & $54 \cdot 8$ & 802 & $54 \cdot 2$ & 778 & 55.4 & \\
\hline$>5$ people & 924 & $32 \cdot 0$ & 433 & $29 \cdot 3$ & 491 & $35 \cdot 0$ & \\
\hline Viral load (copies/ml) & & & & & & & 0.05 \\
\hline Not on ART & 889 & $30 \cdot 8$ & 441 & $29 \cdot 8$ & 448 & 31.9 & \\
\hline On ART, viral load $<1000$ & 1691 & $58 \cdot 6$ & 864 & $58 \cdot 4$ & 827 & $58 \cdot 9$ & \\
\hline On ART, viral load $\geq 1000$ & 304 & 10.5 & 175 & $11 \cdot 8$ & 129 & $9 \cdot 2$ & \\
\hline Highest ever WHO stage & & & & & & & 0.47 \\
\hline 1 & 814 & $28 \cdot 2$ & 435 & $29 \cdot 4$ & 379 & $27 \cdot 0$ & \\
\hline II & 889 & $30 \cdot 8$ & 441 & 29.8 & 448 & 31.9 & \\
\hline III & 998 & 34.6 & 510 & 34.5 & 488 & $34 \cdot 8$ & \\
\hline IV & 183 & $6 \cdot 3$ & 94 & $6 \cdot 4$ & 89 & $6 \cdot 3$ & \\
\hline CD4 count (cells $\left./ \mathrm{mm}^{3}\right)$ & & & & & & & 0.20 \\
\hline$<200$ & 564 & $19 \cdot 6$ & 303 & $20 \cdot 5$ & 261 & $18 \cdot 6$ & \\
\hline$\geq 200$ & 2320 & $80 \cdot 4$ & 1177 & 79.5 & 1143 & $81 \cdot 4$ & \\
\hline Missed doses ART in past month & & & & & & & 0.18 \\
\hline Not on ART & 889 & $30 \cdot 8$ & 441 & $29 \cdot 8$ & 448 & 31.9 & \\
\hline No missed doses ART & 1719 & $59 \cdot 6$ & 906 & $61 \cdot 2$ & 813 & 57.9 & \\
\hline Missed 1+ doses ART & 276 & $9 \cdot 6$ & 133 & $9 \cdot 0$ & 143 & $10 \cdot 2$ & \\
\hline
\end{tabular}

Data are presented as $n$ (column \%). Pearson's $\chi^{2}$ and Wilcoxon rank-sum tests were used to describe differences between participants living with HIV classified as food insecure and those classified as food secure, with food insecurity defined as a report of not having enough food to eat over the past 12 months or having less than three meals per day on average. Statistically significant $p$-values are presented in bold. ART, antiretroviral therapy. 


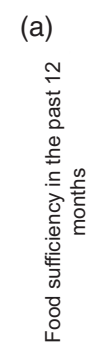

(a)

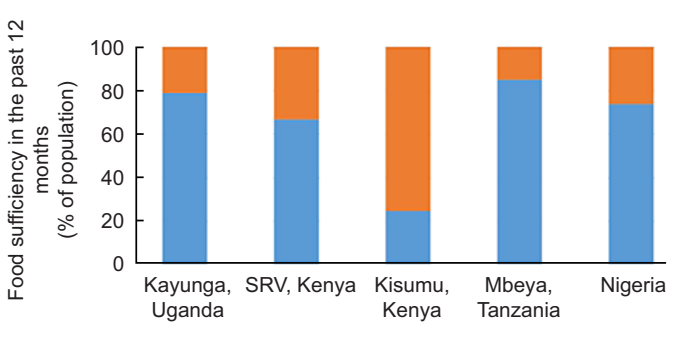

Enough food in past 12 months $=$ Not enough food in past 12 months

(c)

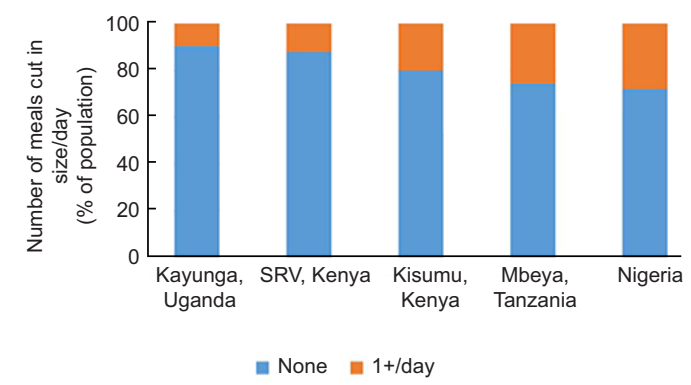

(b)

$p<0 \cdot 01$

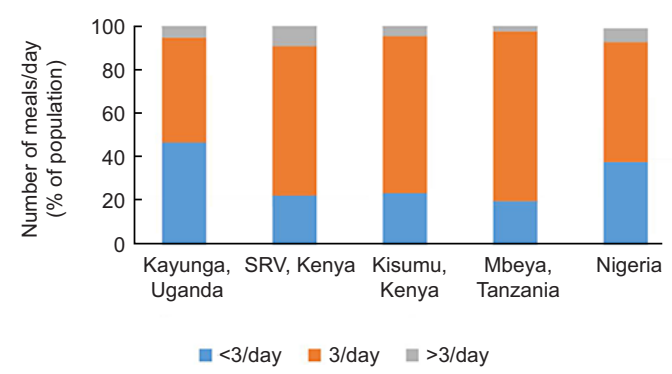

(d) $\quad p<0.01$

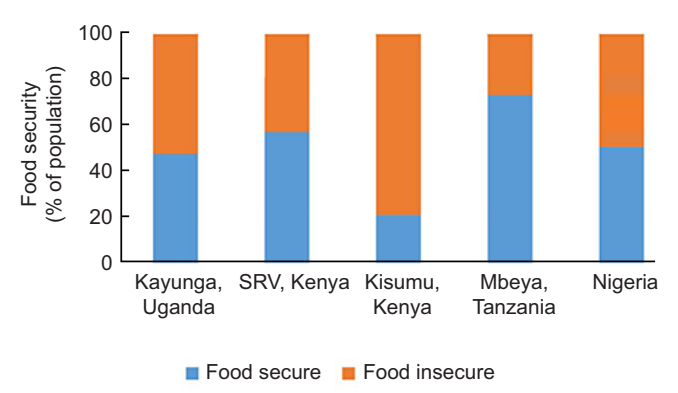

Fig. 1 (colour online) Food security indicators stratified by site in the African Cohort Study

(aPR 1.78, $95 \%$ CI 1.44, 2.21), Kisumu West, Kenya (aPR $3.99,95 \%$ CI $3.22,4.94$ ) or Nigeria (aPR $1.78,95 \%$ CI $1.37,2 \cdot 32)$ as compared to residing in Uganda, was independently associated with not having enough food to eat in the past 12 months. As compared to those with none or some primary education, having a primary or some secondary level education (aPR $0 \cdot 80,95 \%$ CI $0 \cdot 73,0 \cdot 88$ ) or a secondary level education or above (aPR 0.65, $95 \%$ CI 0.57, 0.74 ) was protective against not having enough food to eat in the past 12 months after adjusting for potential confounders. Living with HIV was not significantly associated with not having enough food to eat over the past 12 months (aPR 1.01, 95\% CI 0.90, 1·14).

In the adjusted model for only PLWH, compared to participants 18-24 years old, not having enough food to eat in the past 12 months was more common among those 25-39 years old (aPR 1.40, 95\% CI 1.11, 1.78) and 40-49 years old (aPR 1.52, 95\% CI 1.19, 1.95; Supplementary Table 1). Similar to the model for all participants, living in Kenya (South Rift Valley or Kisumu West) or Nigeria, and having more dependents independently predicted not having enough food to eat over the past 12 months while higher education and being enrolled between 2014 and 2016 compared to 2013 were protective. WHO clinical stage, ART status and CD4 count were not found to be significantly associated with not having enough food to eat in the past 12 months after adjusting for other factors.

\section{Predictors of average number of meals per day}

Among all participants, after adjusting for potential confounders, compared to participants 18-24 years old, those who were 40-49 years (aRR 0.97, $95 \%$ CI 0.94, 1.00) and $50+$ years (aRR 0.95, $95 \%$ CI 0.92, 0.98), male (aRR 0.98, $95 \%$ CI $0.97,1.00$ ) and employed (aRR 0.97, $95 \%$ CI $0.95,0.99)$ had a decreased rate of meals per day (Table 4). Compared to participants from Uganda, participants living in South Rift Valley, Kenya (aRR 1·10, $95 \%$ CI 1.06, 1.14), Kisumu West, Kenya (aRR 1.07, $95 \%$ CI 1.03, 1.11) or Tanzania (aRR 1.07, $95 \%$ CI 1.03, 1.11), and those with a primary or some secondary level education (aRR $1.04,95 \%$ CI $1.02,1.06)$ or a secondary level education or above (aRR 1.06, $95 \%$ CI 1.03, 1.08) as compared to those with none or some primary education had a higher rate of meals per day. There was no significant association between living with HIV and number of meals received per day (aRR 1.01, 95\% CI 0.99, 1.03).

Similarly, PLWH who were older, male and employed had a reduced rate of meals per day, while living in Kenya (South Rift Valley or Kisumu West) or Tanzania compared to Uganda and higher education was associated with an increased rate of meals per day (Supplementary Table 2). ART-experienced PLWH had higher rates of meals per day as compared to ART-naïve participants, irrespective of VL (on ART/VL < 1000 copies/ml aRR 1.04, $95 \%$ CI 1.02, 1.06 and on ART/VL $\geq 1000$ copies/ml aRR 1.03, $95 \%$ CI $1.00,1 \cdot 07)$. 
Table 3 Unadjusted and adjusted analyses of factors associated with not having enough food to eat in the past 12 months among all participants

\begin{tabular}{|c|c|c|c|c|c|}
\hline & \multicolumn{2}{|c|}{ Unadjusted PR (95\% Cl) } & \multicolumn{3}{|c|}{ Adjusted PR $(95 \% \mathrm{Cl})$} \\
\hline & PR & $95 \% \mathrm{Cl}$ & PR & & $95 \% \mathrm{Cl}$ \\
\hline \multicolumn{6}{|l|}{ HIV status } \\
\hline People living with HIV & 1.05 & $0.93,1.18$ & 1.01 & & $0.90,1.14$ \\
\hline People living without HIV & \multicolumn{2}{|c|}{ Ref } & \multicolumn{3}{|c|}{-} \\
\hline \multicolumn{6}{|l|}{ Age (years) } \\
\hline $18-24$ & \multicolumn{2}{|c|}{ Ref } & \multicolumn{3}{|c|}{ - } \\
\hline $25-39$ & 1.70 & $1.38,2.09$ & 1.35 & & $1.10,1.64$ \\
\hline $40-49$ & 1.91 & $1.55,2.36$ & 1.58 & & $1.28,1.94$ \\
\hline $50+$ & 1.87 & $1.50,2.33$ & 1.33 & & $1.07,1.65$ \\
\hline \multicolumn{6}{|l|}{ Sex } \\
\hline Male & 1.03 & $0.94,1.13$ & 1.01 & & $0.93,1.10$ \\
\hline Female & \multicolumn{2}{|c|}{ Ref } & \multicolumn{3}{|c|}{ - } \\
\hline \multicolumn{6}{|l|}{ Study site } \\
\hline Kayunga, Uganda & \multicolumn{2}{|c|}{ Ref } & \multicolumn{3}{|c|}{ - } \\
\hline South Rift Valley, Kenya & 1.58 & $1.33,1.87$ & 1.78 & & $1.44,2.21$ \\
\hline Kisumu West, Kenya & 3.59 & $3.07,4.20$ & 3.99 & & $3.22,4.94$ \\
\hline Mbeya, Tanzania & 0.71 & $0.56,0.90$ & 0.87 & & $0.66,1.15$ \\
\hline Abuja and Lagos Nigeria & 1.25 & $0.99,1.56$ & 1.78 & & $1.37,2.32$ \\
\hline \multicolumn{6}{|l|}{ Year enrolled in AFRICOS } \\
\hline 2013 & \multicolumn{2}{|c|}{ Ref } & \multicolumn{3}{|c|}{-} \\
\hline 2014 & $1 \cdot 19$ & $0.99,1.42$ & 0.80 & & $0.66,0.97$ \\
\hline 2015 & $1 \cdot 10$ & $0.92,1.33$ & 0.77 & & $0.64,0.93$ \\
\hline 2016 & 1.25 & $1.04,1.51$ & 0.79 & & $0.65,0.96$ \\
\hline 2017 & 0.93 & $0.73,1.19$ & 0.96 & & $0.75,1.22$ \\
\hline 2018 & 0.33 & $0.18,0.60$ & 0.42 & & $0.23,0.77$ \\
\hline $2019 / 2020$ & 0.39 & $0.23,0.66$ & 0.49 & & $0.29,0.83$ \\
\hline \multicolumn{6}{|l|}{ Marital status } \\
\hline Not married & \multicolumn{2}{|c|}{ Ref } & \multicolumn{3}{|c|}{-} \\
\hline Married & 1.19 & $1.08,1.31$ & 0.91 & & $0.83,1.00$ \\
\hline \multicolumn{6}{|l|}{ Education } \\
\hline None or some primary & \multicolumn{2}{|c|}{ Ref } & \multicolumn{3}{|c|}{ - } \\
\hline Primary or some secondary & 0.73 & $0.66,0.80$ & 0.80 & & $0.73,0.88$ \\
\hline Secondary and above & 0.58 & $0.51,0.65$ & 0.65 & & $0.57,0.74$ \\
\hline Employment status & & & & & \\
\hline Not employed & & & & - & \\
\hline Employed & 0.68 & $0.62,0.76$ & 0.98 & & $0.86,1.12$ \\
\hline Currently a farmer & & & & & \\
\hline No & & & & - & \\
\hline Yes & 0.70 & $0.59,0.85$ & 0.96 & & $0.77,1.21$ \\
\hline Total no. people in household & & & & & \\
\hline$\leq 6$ & & & & - & \\
\hline$>6$ & 1.08 & $0.97,1.21$ & 0.98 & & $0.87,1.09$ \\
\hline No. of dependents & & & & & \\
\hline$<2$ people & & & & - & \\
\hline 2-5 people & 1.63 & $1.35,1.97$ & 1.27 & & $1.06,1.52$ \\
\hline$>5$ people & 1.93 & $1.60,2.34$ & 1.41 & & $1.16,1.71$ \\
\hline
\end{tabular}

Generalised linear models with a Poisson distribution and robust error variances were used to estimate unadjusted and adjusted prevalence ratios (aPR) and $95 \% \mathrm{Cl}$ for associations between pre-specified factors of interest and not having enough food to eat in the past 12 months among all participants. Statistically significant association is in bold. AFRICOS, African Cohort Study.

\section{Predictors of food insecurity based on the combined index}

After adjusting for potential confounding factors, compared to participants 18-24 years old, food insecurity as assessed using the combined index, was more common among those 25-39 years (aPR 1.26, $95 \%$ CI 1.08, 1.46), 40-49 years (aPR 1.41, $95 \%$ CI 1.20, 1.65) and 50+ years (aPR 1.35, $95 \%$ CI 1.15, 1.59; Table 5). As compared to having less than two dependents, having 2-5 dependents (aPR 1.14, 95\% CI 1.01, 1.30) or more than five dependents (aPR 1.17, $95 \%$ CI 1.02, 1.35) was independently associated with food insecurity. Residing in Kisumu
West, Kenya (aPR 1.63, $95 \%$ CI 1.42, 1.87) or Nigeria (aPR $1 \cdot 20,95 \%$ CI $1.01,1.41$ ) as compared to residing in Uganda, was independently associated with food insecurity while residing in Tanzania (aPR 0.60, $95 \%$ CI 0.50, $0 \cdot 72$ ) was protective against food insecurity. As compared to those with none or some primary education, having a primary or some secondary level education (aPR $0 \cdot 82$, $95 \%$ CI $0.76,0.88)$ or a secondary level education or above (aPR 0.73, $95 \%$ CI 0.66, 0.81) was protective against food insecurity. Living with HIV was not a predictor of food insecurity as assessed by the combined index (aPR $1 \cdot 01,95 \%$ CI $0 \cdot 92,1 \cdot 10)$. 
Table 4 Unadjusted and adjusted analyses of factors associated with number of meals per day among all participants

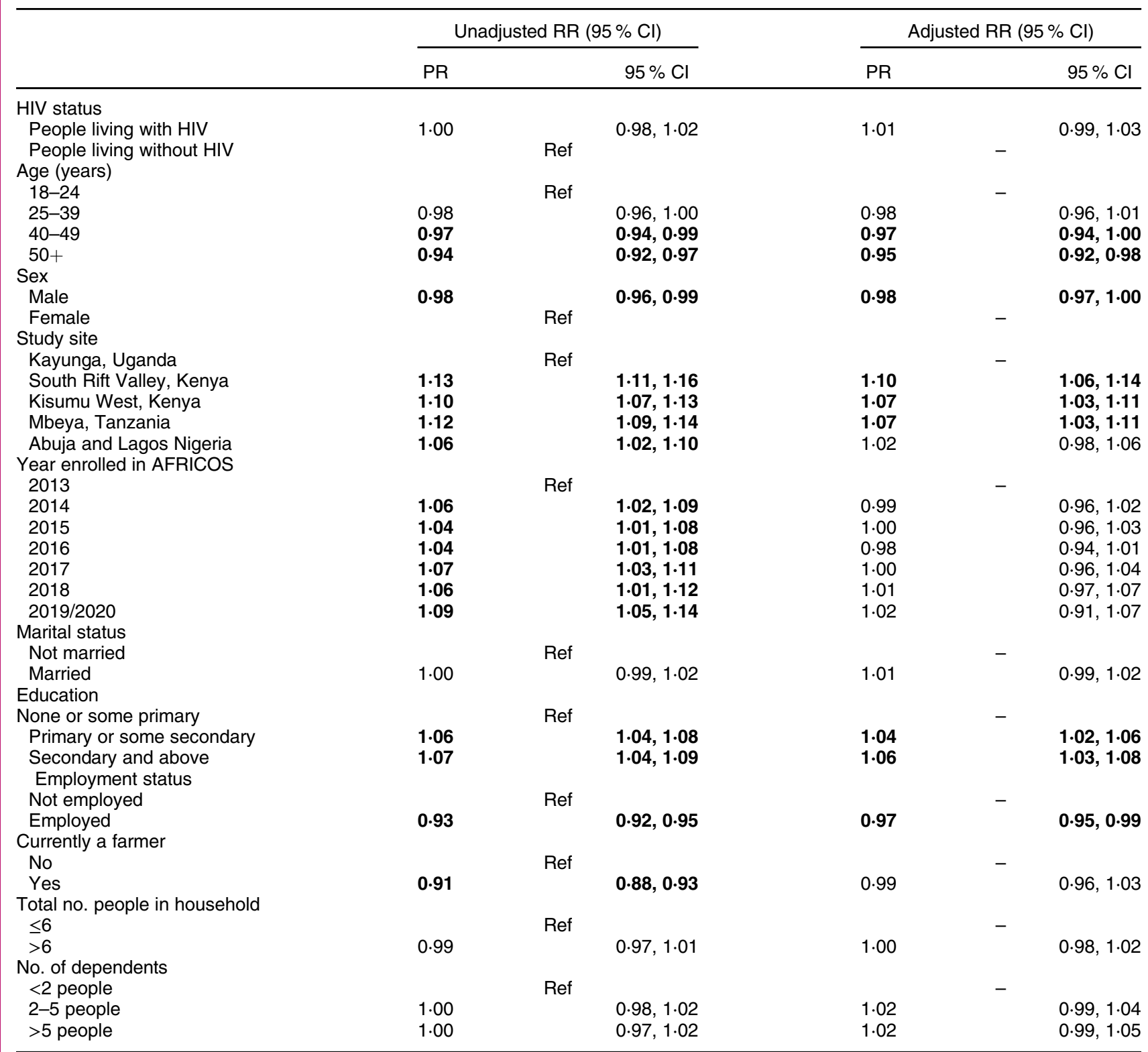

Poisson regression models were used to estimate unadjusted and adjusted rate ratios for associations between pre-specified factors and the number of meals per day among all participants. Statistically significant association in bold. AFRICOS, African Cohort Study.

After adjusting for potential confounding factors, among PLWH, compared to participants 18-24 years old, food insecurity was more common among those 25-39 years old (aPR 1.31, $95 \%$ CI 1.09, 1.57), 40-49 years (aPR 1.40, 95\% CI 1.16, 1.69) and 50+ years (aPR 1.39, 95 \% CI 1.14, 1.69; Supplementary Table 3). As compared to having less than two dependents, having 2-5 dependents (aPR 1.19, 95\% CI 1.03, 1.37) or more than five dependents (aPR 1.21, $95 \%$ CI 1.03 , 1.41) was independently associated with food insecurity among PLWH. Residing in Kisumu West, Kenya (aPR $1.83,95 \%$ CI $1.56,2.15$ ) or Nigeria (aPR 1.30, $95 \%$ CI $1.08,1.57)$ as compared to residing in Uganda, was independently associated with food insecurity while residing in Tanzania (aPR 0.65, $95 \%$ CI $0.53,0.80$ ) was protective. Compared to those with none or some primary education, having a primary or some secondary level education (aPR 0.81, $95 \%$ CI $0.75,0.88)$ or a secondary level education or above (aPR $0.73,95 \%$ CI $0.66,0.82$ ) was protective against food insecurity. ART-experienced PLWH were significantly more likely to be food secure irrespective of VL compared to ART-naïve PLWH (on ART/ $\mathrm{VL}<1000$ copies/ml $v$. ART naïve, aPR 0.90, $95 \%$ CI 0.82, 0.99; on ART/VL $\geq 1000$ copies/ml $v$. ART naïve, aPR $0 \cdot 86,95 \%$ CI $0.74,0.99)$. WHO clinical stage and CD 4 count were not significantly associated with food insecurity in the multivariable analysis. 
Table 5 Unadjusted and adjusted analyses of factors associated with food insecurity among all participants

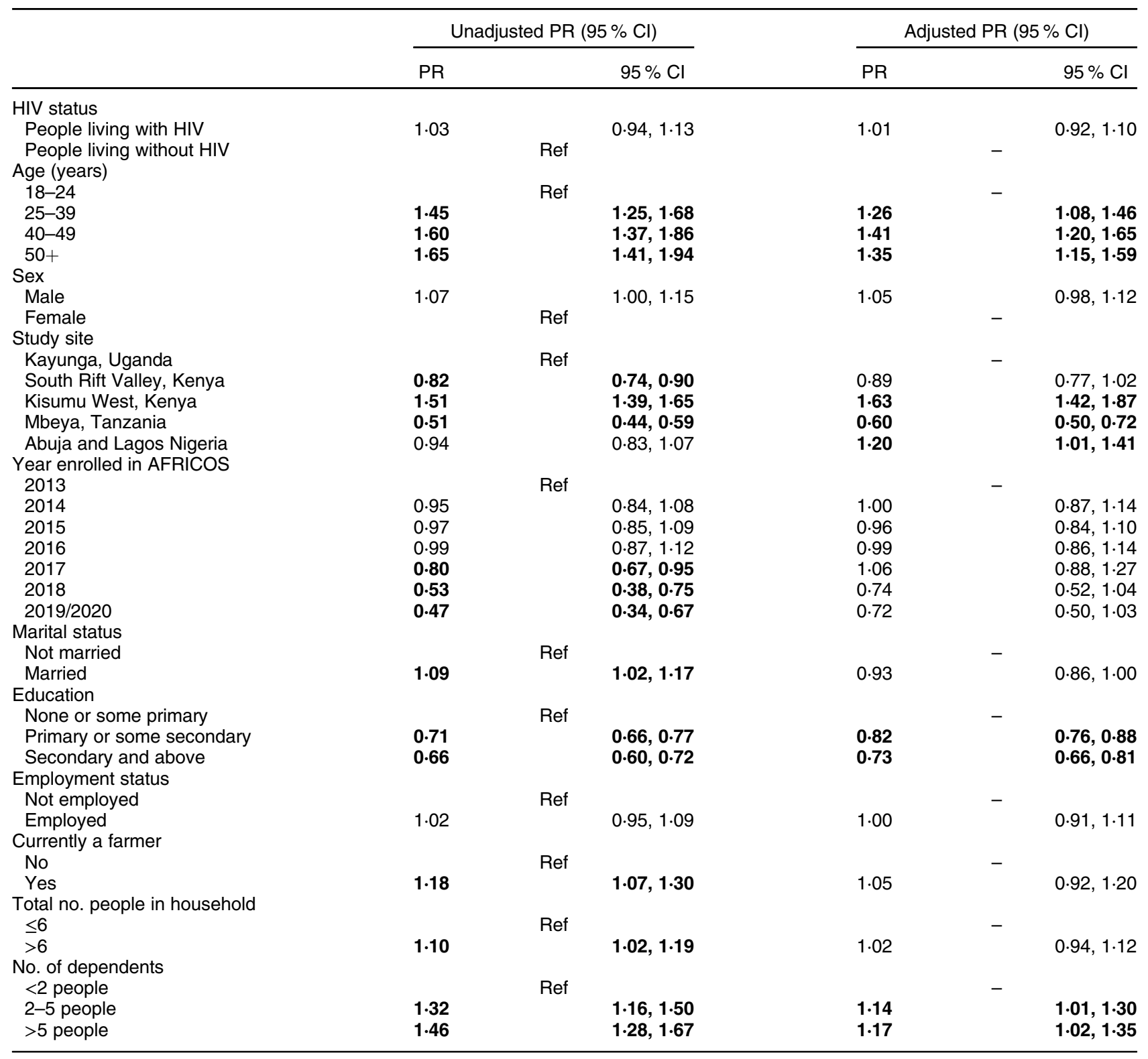

Generalised linear models with a Poisson distribution and robust error variances were used to estimate unadjusted and adjusted prevalence ratios (aPR) and $95 \% \mathrm{CI}$ for associations between pre-specified factors of interest and food insecurity, defined as a report of not having enough food to eat over the past 12 months or having less than three meals per day on average, among all participants. Statistically significant association is indicated in bold. AFRICOS, African Cohort Study.

\section{Discussion}

Between one-fifth and one-third of participants in our study recorded at least one of three food insecurity metrics with significant inter-site differences. As defined by the combined index, nearly half of the participants reported food insecurity, underscoring a large unmet need for strategies to ensure adequate food supply to people with and without HIV in SSA. This finding is generally consistent with prior reports of high but variable prevalence of food insecurity in SSA ranging from 19.5 to $100 \%{ }^{(11,18,20-28,32-38)}$. In Western Kenya, $32 \%$ of patients attending diabetes clinics were found to be food insecure ${ }^{(20)}$, while studies from other parts of Kenya found much higher rates of food insecurity of $85 \%$ in urban slum residents following post-election crises ${ }^{(37)}$ and $100 \%$ in a very small population of PLWH ${ }^{(24)}$. Available studies from Uganda have also demonstrated high levels of food insecurity of 75.4$93 \%$ in the general population ${ }^{(23,38)}$ and among predominantly ART-naïve PLWH ${ }^{(26,28)}$. Among ART-naïve PLWH in Tanzania, $52.2 \%$ were found to be food insecure ${ }^{(27)}$. The prevalence of food insecurity in various studies conducted in largely rural and low-income Nigerian households ranged from $40 \cdot 8$ to $70 \%(11,21,32,33,35)$. In Southern Ethiopia, a study conducted in predominantly stable, ART-experienced PLWH found a relatively lower prevalence of food insecurity of $19.5 \%(36)$. In addition to the differences in the 
methodologies used to assess food security, characteristics of the study population, sample size, environmental and sociopolitical issues may account for the disparity in these studies. Several tools are available for the measurement of food insecurity ${ }^{(39)}$. Most of the previous studies in SSA used the Household Food Insecurity Access Scale, an experiencebased tool with a 30 -day recall ${ }^{(24,26,28,37,40)}$. Some studies in SSA have used the food security index ${ }^{(11,33)}$ or different questionnaire-based tools ${ }^{(20,22)}$, while others used three or single-question food security indicators over a 12-month recall similar to our methods ${ }^{(27,41)}$. It has been shown that all the methods have advantages and inherent measurement errors, and no single tool can account for all the dimensions of food security ${ }^{(39)}$.

In this study, similar socio-demographic factors consistently predicted food insecurity whether assessed by singular metrics or the combined index. Also, the sociodemographic predictors of food insecurity were similar for all participants and the subgroup of PLWH, who constituted the majority of the study population. The predictors of food insecurity among all participants, as defined by the combined index, are largely consistent with findings of prior studies conducted in SSA that identified low educational status $^{(11,21,24,33-35,40)}$ age $^{(11,21,24)}$ and a high number of dependents $^{(33)}$ as predictors of food insecurity. A high level of education is likely to enable people to access better job opportunities, attract higher income and make more appropriate decisions regarding food production and/or consumption. Older age may be associated with a decline in physical strength leading to reduced farming activities or limited ability to engage in relatively better-paying jobs, thereby predisposing to food insecurity ${ }^{(42,43)}$. We also observed that the geographical location of participants impacted food security, whether assessed by single metrics or the combined index. Compared to participants from Uganda, living in Kisumu West, Kenya was independently predictive of food insecurity in our cohort. Few studies have investigated the impact of geographical location on food insecurity. In tandem with our report, a previous study in Brazil observed that residing in the North and Northeastern regions of the country was predictive of food insecurity ${ }^{(44)}$. Among all participants, married persons were significantly more likely to be food secure. This observation was the only sociodemographic predictor of food insecurity seen in the mixed population but not in the PLWH subgroup in the adjusted model. In Western Ethiopia, being single predicted food insecurity in $\mathrm{PLWH}^{(40)}$. It is possible that the care and support provided by a spouse could positively impact food access and the availability of prepared meals at home.

Other factors commonly associated with food insecurity in previous studies are low household income, large family size, unemployment, occupation, temporal changes/year and limited involvement in farming ${ }^{(11,21,33-35,40)}$. Contrary to some previous studies, employment status, being a farmer and size of household were not independent predictors of food insecurity defined by the combined index in our cohort. Nevertheless, we made an interesting observation that being employed rather predicted having a lower number of meals per day on average among all participants. It is understandable that being at work may lead to missing meals on some days. However, this cannot be stretched too far as employment status neither impacted report of not having enough food to eat over the past 12 months nor food insecurity assessed by the combined index. Although the year of enrolment did not emerge as an independent predictor of food insecurity in our cohort, it proved to be a significant factor associated with the report of not having enough food to eat over the past 12 months in all participants and in the subgroup of PLWH. The impact of temporal changes on food insecurity has been previously documented $^{(44)}$. Multiple factors may be responsible such as the impact of seasonal/climatic variations on agricultural activities and the role of socio-political crises on food access. Due to the variability of occupation and income/ national currencies in our population, we did not specifically examine the relationship between food insecurity and various occupations or household income.

Although a household size of more than six and surprisingly being a farmer were significantly associated with food insecurity in unadjusted models, these associations were not statistically significant after adjusting for potential confounders. While we did not find any significant association between sex and food insecurity as defined by the combined index, we observed that being male was significantly associated with fewer meals per day among all participants and in the subgroup of PLWH. The association of male sex with fewer meals per day may be because males are more likely to be employed and may thus need to miss a meal due to being at work. Although being male was significantly associated with food insecurity compared to female sex among PLWH in a previous study in Western Ethiopia ${ }^{(40)}$, another study in Southern Ethiopia identified being female as a predictor of food insecurity in PLWH ${ }^{(36)}$. This disparity was observed even though both studies assessed food insecurity using the same tool. A systematic review of the effect of sex on food security among PLWH receiving ART showed higher odds of food insecurity among female PLWH than their male counterparts, especially in low and middleincome countries where women lack control over resources and household food allocation decision-making ${ }^{(45)}$.

There is a paucity of studies comparing the prevalence of food insecurity between PLWH and those without HIV. Surprisingly, this study revealed that PLWH were not disproportionately affected by food insecurity both in the combined index and for the single metrics, contrary to the traditional view that HIV infection predisposes to food insecurity $^{(14,15)}$. A possible reason for our observation is that the PLWH in our study were engaged in care and the majority were on suppressive ART, hence were unlikely to be at substantial risk of decreased food production efficiency and higher out-of-pocket expenditure on healthcare. Moreover, this study predominantly spans an era of 
improved access to HIV care and treatment during which a relatively smaller proportion of PLWH presented with advanced disease, compared to studies conducted predominantly in the era of limited access to care with a substantial proportion of PLWH manifesting advanced disease ${ }^{(26-28)}$.

Nevertheless, we conducted more focussed analyses in the subgroup of PLWH to better understand the predictors of food insecurity in the population, beyond socio-demographic factors which themselves are similar between PLWH and the mixed population in this study. In terms of HIV care/outcome variables, we observed that ART-experienced PLWH were significantly more likely to be food secure irrespective of their viral suppression status compared to ART-naïve PLWH. However, advanced HIV disease was not significantly associated with food insecurity. Previous studies have demonstrated an inverse relationship between food insecurity and key HIV care/outcome variables $^{(17-19)}$. An inverse relationship between food insecurity and ART access has been documented in SSA ${ }^{(17)}$. For example, food insecurity was shown to be a barrier to ART in PLWH yet to initiate treatment and a key contributor to incomplete adherence among individuals on $\mathrm{ART}^{(17)}$. Studies conducted in the USA have reported a significant association between food insecurity and poor virologic response in ART-experienced PLWH after controlling for potential confounders ${ }^{(46,47)}$. Improved food security seen in the ART-experienced PLWH in this study is not surprising considering that the attendant improved health and wellbeing is likely to have downstream consequences on psychosocial and economic factors known to improve food access. Sustained improvement of access to ART could potentially be exploited as a strategy for improving food security in SSA, especially among PLWH.

Contrary to our observation, advanced HIV disease as evidenced by WHO clinical stage III/IV disease or low CD4 count predicted food insecurity in Southern Ethiopia and North America ${ }^{(36,48,49)}$. Some previous studies have demonstrated heavy household expenditure on healthcare in PLWH with advanced disease thereby predisposing to poverty and invariably food insecurity ${ }^{(50,51)}$. In another study in Western Ethiopia, the relationship between food insecurity and WHO clinical stage was inconsistent on multivariate analysis ${ }^{(40)}$. Beyond any potential association with advanced disease, the two-way relationship between HIV-related mortality and food insecurity highlights the critical need to address food security challenges in $\mathrm{PLWH}^{(19,52)}$. On a broader perspective, the negative impact of food insecurity on health outcomes has been documented for several diseases other than $\mathrm{HIV}^{(53,54)}$. Integrating food security interventions into HIV care/treatment programs appears to be a pragmatic step towards improving the health of PLWH. However, as recommended in a previous study, policies and interventions aimed at enhancing food security in SSA should target vulnerable groups broadly, rather than solely targeting those directly affected by $\mathrm{HIV}^{(52)}$.
The limitations of these analyses are acknowledged. Our measurement of food insecurity was not based on one of the more commonly used validated tools such as Household Food Insecurity Access Scale and the food security index, which makes direct comparison with a number of prior studies challenging. As applicable to several food insecurity survey tools, our method is subjective and vulnerable to recall social desirability bias. Also, caution should be exercised when generalising our findings for two major reasons. First, the study participants were enrolled during clinic visits so it may represent a disproportionate sample of vulnerable individuals at increased risk of food insecurity. Secondly, our study population comprised predominantly PLWH and a smaller proportion of adults without HIV, which is not the typical picture in the general population. The study participants were enrolled over a period of 7 years during which diverse seasonal and socio-political factors could have impacted food production and access in Africa thereby constituting a potential limitation to these analyses. Despite these limitations, the strengths of this study are worthy of note. We examined food insecurity among a large cohort in four African countries, highlighting significant inter-country differences in food insecurity and invariably filling some gaps in previous studies. We were able to control for multiple potential confounders, including socio-demographic variables and year of enrolment, some of which were not adjusted for in some previous studies. The potential impact of seasonal/climatic variations on food insecurity was partly controlled by the inclusion of the year of enrolment in the models. While the impact of our study limitations on the high prevalence of food insecurity and the lack of association with HIV is worthy of consideration, the fact that the majority of previous studies in SSA have either reported similarly high or much higher burden of food insecurity in the general population, and in PLWH lays credence to our observations. These analyses have also provided findings that argue for further studies on the relationship between HIV outcomes and food insecurity in the era of improved access to care and treatment.

In conclusion, we found a high prevalence of food insecurity in four African countries, with significant inter-country variability. Surprisingly, the prevalence of food insecurity among PLWH was similar to that of people without HIV. This study revealed that age, education, number of dependents, country of residence and marital status were independent predictors of food insecurity assessed using a combined index. The sociodemographic predictors of food insecurity were similar between the mixed population and the subgroup of PLWH. Irrespective of VL suppression status, ART-experienced PLWH were significantly more likely to be food secure compared to ART-naïve populations. On the other hand, no significant association was demonstrated between advanced HIV disease and food insecurity. The high prevalence of food insecurity in SSA is concerning, especially in the setting of COVID-19- 
induced socio-economic crises and reduction in food security. Moreover, a high level of food insecurity remains a threat to the attainment of Sustainable Development Goals in Africa. Increased access to education for the entire population and providing social support for older populations could potentially improve food security in SSA. Given the strong extended family system in Africa, in addition to intensified family planning sensitisation, the economic empowerment of eligible family members will reduce the pressure on household heads with a potentially favourable downstream impact on food security. Improved access to ART is a pragmatic strategy for promoting food security in PLWH. African governments should prioritise agricultural policies that support food production, distribution and access. We recommend further prospective cohort studies to investigate the relationship between HIV outcomes and food insecurity.

\section{Acknowledgments}

We thank the study participants, local implementing partners and hospital leadership at Kayunga District Hospital, Kericho District Hospital, AC Litein Mission Hospital, Kapkatet District Hospital, Tenwek Mission Hospital, Kapsabet District Hospital, Nandi Hills District Hospital, Kisumu West District Hospital, Mbeya Zonal Referral Hospital, Mbeya Regional Referral Hospital, Defence Headquarters Medical Center and the $68^{\text {th }}$ Nigerian Army Reference Hospital.

We would also like to thank the AFRICOS Study Group from the US Military HIV Research Program Headquarters team: Danielle Bartolanzo, Alexus Reynolds, Katherine Song, Mark Milazzo, Leilani Francisco, Shauna Mankiewicz, Steven Schech, Alexandra Golway, Badryah Omar, Tsedal Mebrabtu, Elizabeth Lee, Kimberly Bohince, Ajay Parikh, Jaclyn Hern, Emma Duff, Kara Lombardi, Michelle Imbach and Leigh Anne Eller; from the AFRICOS Uganda team: Michael Semwogerere, Prossy Naluyima, Godfrey Zziwa, Allan Tindikahwa, Hilda Mutebe, Cate Kafeero, Enos Baghendaghe, William Lwebuge, Freddie Ssentogo, Hellen Birungi, Josephine Tegamanyi, Paul Wangiri, Christine Nabanoba, Phiona Namulondo, Richard Tumusiime, Ezra Musingye, Christina Nanteza, Joseph Wandege, Michael Waiswa, Evelyn Najiuma, Olive Maggaga, Isaac Kato Kenoly and Barbara Mukanza; from the AFRICOS South Rift Valley, Kenya team: Rither Langat, Aaron Ngeno, Lucy Korir, Raphael Langat, Francis Opiyo, Alex Kasembeli, Christopher Ochieng, Japhet Towett, Jane Kimetto, Brighton Omondi, Mary Leelgo, Michael Obonyo, Linner Rotich, Enock Tonui, Ella Chelangat, Joan Kapkiai, Salome Wangare, Zeddy Bett Kesi, Janet Ngeno, Edwin Langat, Kennedy Labosso, Joshua Rotich, Leonard Cheruiyot, Enock Changwony, Mike Bii, Ezekiel
Chumba, Susan Ontango, Danson Gitonga, Samuel Kiprotich, Bornes Ngtech, Grace Engoke, Irene Metet, Alice Airo and Ignatius Kiptoo; from the AFRICOS Kisumu, Kenya team: Valentine Sing'oei, Winne Rehema, Solomon Otieno, Celine Ogari, Elkanah Modi, Oscar Adimo, Charles Okwaro, Christine Lando, Margaret Onyango, Iddah Aoko, Kennedy Obambo, Joseph Meyo and George Suja; from the AFRICOS Abuja, Nigeria Group: Michael Iroezindu, Yakubu Adamu, Nnamdi Azuakola, Mfreke Asuquo, Abdulwasiu Bolaji Tiamiyu, Afoke Kokogho, Samirah Sani Mohammed, Ifeanyi Okoye, Sunday Odeyemi, Aminu Suleiman, Lawrence C. Umeji, Onome Enas, Miriam Ayogu, Ijeoma Chigbu-Ukaegbu, Wilson Adai, Felicia Anayochukwu Odo, Rabi Abdu, Roseline Akiga, Helen Nwandu, Chisara Sylvestina Okolo, Ogundele Taiwo, Otene Oche Ben, Nicholas Innocent Eigege, Tony Ibrabim Musa, Juliet Chibuzor Joseph, Ndubuisi C. Okeke; from the AFRICOS Lagos, Nigeria Group: Zabra Parker, Nkechinyere Elizabeth Harrison, Uzoamaka Concilia Agbaim, Olutunde Ademola Adegbite, Ugochukwu Linus Asogwa, Adewale Adelakun, Chioma Ekeocha, Victoria Idi, Rachel Eluwa, Jumoke Titilayo Nwalozie, Igiri Faith, Blessing Irekpitan Wilson, Jacinta Elemere, Nkiru Nnadi, Francis Falaju Idowu, Ndubuisi Rosemary, Amaka Natalie Uzoegwu, Theresa Owanza Obende, Ifeoma Lauretta Obilor, Doris Emekaili, Edward Akinwale and Inalegwu Ochai; from the AFRICOS Mbeya, Tanzania team: Lucas Maganga, Samoel Khamadi, John Njegite, Connie Lueer, Abisai Kisinda, Jaquiline Mwamwaja, Faraja Mbwayu, Gloria David, Mtasi Mwaipopo, Reginald Gervas, Doroth Mkondoo, Nancy Somi, Paschal Kiliba, Gwamaka Mwaisanga, Johnisius Msigwa, Hawa Mfumbulwa, Peter Edwin, Willyhelmina Olomi.

\section{Financial Support}

This work was supported by the President's Emergency Plan for AIDS Relief via a cooperative agreement between the Henry M. Jackson Foundation for the Advancement of Military Medicine, Inc. and the U.S. Department of Defense [W81XWH-18-2-0040].

\section{Conflict of Interest}

The authors have no conflicts of interest to disclose.

\section{Author Contributions}

JAA and CSP designed the research study. JO, JM, EB, HK and $\mathrm{MI}$ collected the research data. AE and ND analysed the data. CCO, RUN, MI, AE, ND and TAC wrote the paper. All authors have read and approved the final manuscript. 


\section{Ethics of Human Subject Participation}

This study was conducted according to the guidelines laid down in the Declaration of Helsinki and all procedures involving research study participants were approved by the institutional review boards of the Walter Reed Army Institute of Research, Silver Spring, MD, USA; Makerere University School of Public Health, Kampala, Uganda; Kenya Medical Research Institute, Nairobi, Kenya; Tanzania National Institute of Medical Research, Mbeya, Tanzania; and Nigerian Ministry of Defence, Abuja, Nigeria. Written informed consent was obtained from all participants.

\section{Disclaimer}

The views expressed are those of the authors and should not be construed to represent the positions of the US Army, the Department of Defense or the Department of Health and Human Services. The investigators have adhered to the policies for protection of human subjects as prescribed in AR-70.

\section{Supplementary material}

To view supplementary material for this article, please visit https://doi.org/10.1017/\$136898002100361X

\section{References}

1. Food and Agriculture Organization (FAO) of the United Nations (2016) Policy Brief: Food Security; available at http://www.fao. org/fileadmin/templates/faoitaly/documents/pdf/pdf_Food_ Security_Cocept_Note.pdf (accessed 02 October 2018).

2. FAO, IFAD, UNICEF, WFP and WHO (2018) The State of food security and nutrition in the World 2018: Building Climate Resilience for Food Security and nutrition; available at http://www.fao.org/3/i9553en/i9553en.pdf (accessed 27 March 2019).

3. FAO, UN Economic Commission for Africa. (2018) Africa Regional Overview of Food Security and Nutrition: Addressing the Threat from Climate Variability and Extremes; available at https://reliefweb.int/sites/reliefweb. int/files/resources/ca2710en.pdf (accessed 28 October 2020).

4. Rezende Machado de Sousa L, Saint-Ville A, SamayoaFigueroa L et al. (2019) Changes in food security in Latin America from 2014 to 2017. Food Sec 11, 503-513.

5. FAO, UNICEF, WHO, WFFP (2018) Asia and the Pacific Regional Overview of Food Security and Nutrition: Accelerating Progress Towards SDGs; available at https:// reliefweb.int/sites/reliefweb.int/files/resources/CA0950EN. pdf (accessed 28 October, 2020).

6. Food and Agricultural Organization of the United Nations (2018) Food Insecurity and Poverty, a Major Challenge to Meeting SDGs Target 2.1 in Sub-Saharan Africa; available at http://www.fao.org/africa/news/detail-news/en/c/471980 (accessed 13 December 2018).

7. United Nations (2006) Millennium Development Goals Report, 2006. United Nations, New York; available at: https://www.un.
org/zh/millenniumgoals/pdf/MDGReport2006.pdf (accessed 21 September 2020).

8. Alabi RA (2003) Human capital as determinant of technical insufficiency of cocoa based agro-forestry system. Food Agric Environ 1, 277-281.

9. Choi SK, Fram MS \& Frongillo EA (2017) Very low food security in U.S. households is predicted by complex patterns of health, economics, and service participation. J Nutr 147, 1992-2000.

10. Chinnakali P, Upadhyay RP, Shokeen D et al. (2014) Prevalence of household-level food insecurity and its determinants in an urban resettlement colony in north India. J Health Popul Nutr 32, 227-236.

11. Mustapha M, Kamaruddin RB \& Dewi S (2018) Assessing the food security determinants among rural households in Kano, Nigeria. J Agric Econ Rural Dev 4, 494-500.

12. Mohammed M \& Dlamini T (2018) Predictors of food insecurity in Eswatini: lessons from the 2015/16 El Niño induced drought. Afr Rev Econ Financ 10, 69-96.

13. The Joint United Nations Programme on HIV/AIDS (UNAIDS) (2019) Global HIV and AIDS Statistics Fact Sheet; available at https://www.unaids.org/sites/default/ files/media_asset/UNAIDS_FactSheet_en.pdf (accessed 20 September 2020).

14. Food and Agricultural Organization of the United Nations (2001) Committee on World Food Security: The Impact of HIV/AIDS on Food Security; available at http://www.fao. org/docrep/meeting/003/Y0310E.htm (accessed 13 December 2018).

15. Oyekale AS \& Adeoti AI (2010) HIV/AIDS and efficiency of food production in the rainforest belt of Nigeria.J Sustainable Dev Afr 12, 72-82.

16. Rollins N (2007) Food insecurity- a risk factor for HIV infection. Plos Med 4, 301.

17. Young S, Wheeler AC, McCoy SI \& Weiser SD O A review of the role of food insecurity in adherence to care and treatment among adult and pediatric populations living with HIV and AIDS. AIDS Behav 18, 505-515.

18. Hong SY, Fanelli TJ, Jonas A et al. (2014) Household food insecurity associated with antiretroviral therapy adherence among HIV-infected patients in Windhoek, Namibia. J Acquir Immune Defic Syndr 67, 115-22.

19. Anema A, Vogenthaler N, Frongillo EA, Kadiyala S \& Weiser SD (2009) Food insecurity and HIV/AIDS: current knowledge, gaps, and research priorities. Curr HIV/AIDS Rep 6, 224-231.

20. Cheng S, Kamano J, Kirui NK et al. (2013) Prevalence of food insecurity in patients with diabetes in western Kenya. Diabet Med 30, 215-222.

21. Oyebanjo OA, Ambali OI \& Akerele EO (2013) Determinants of food security status and incidence of food insecurity among rural farming households in Ijebu division of Ogun state Nigeria. J Agric Sci Environ 13, 92-103.

22. Napier C, Oldewage-Theron W \& Makhaye B (2018) Predictors of food insecurity and coping strategies of women asylum seekers and refugees in Durban, South Africa. Agric Food Secur 7, 1-9.

23. Yikii F, Turyahabwe N \& Bashaasha B (2017) Prevalence of household food insecurity in wetland adjacent areas of Uganda. Agric Food Secur 6, 63.

24. Nagata JM, Magerenge RO, Young SL et al. (2012) Social determinants, lived experiences, and consequences of household food insecurity among persons living with HIV/ AIDS on the shore of Lake Victoria, Kenya. AIDS Care 24, $728-736$.

25. Masa R, Chowa G \& Nyirenda V (2017) Prevalence and predictors of food insecurity among people living with HIV enrolled in antiretroviral therapy and livelihood programs in two rural Zambian hospitals. Ecol Food Nutr $\mathbf{5 6}$, $256-276$ 
26. Weiser SD, Tsai AC, Gupta R et al. (2012) Food insecurity is associated with morbidity and patterns of healthcare utilization among HIV-infected individuals in a resource-poor setting. AIDS 26, 67-75.

27. Semali IA, Edwin T \& Mboera LE (2011) Food insecurity and coping strategies among people living with HIV in Dar es Salaam, Tanzania. Tanzan J Health Res 13, 86-94.

28. Tsai AC, Bangsberg DR, Emenyonu N et al. (2011) The social context of food insecurity among persons living with HIV/ AIDS in rural Uganda. Soc Sci Med 73, 1717-1724.

29. Amewu S, Asante S, Pauw K \& Thurlow J (2020) The economic costs of COVID-19 in sub-Saharan Africa: insights from a simulation exercise for Ghana. Eur J Dev Res; available at https://pubmed.ncbi.nlm.nih.gov/33144762/ (accessed 01 May 2021).

30. Dear N, Duff E, Esber A et al. (2021) Transient reductions in HIV clinic attendance and food security during the COVID-19 pandemic for people living with HIV in four African countries. Clin Infect Dis 27. doi: $10.1093 / \mathrm{cid} /$ ciab379.

31. Ake JA, Polyak CS, Crowell TA et al. (2019) Noninfectious comorbidity in the African Cohort Study. Clin Infect Dis 69, 639-647.

32. Orewa SI \& Iyangbe CO (2009) The food insecurity profile among the rural and low-income urban dwellers in Nigeria. Am-Euras J Sci Res 4, 302-307.

33. Akerele D, Momoh S, Aromolaran AB et al. (2013) Food insecurity and coping strategies in South-West Nigeria. Food Sec 5, 407-414

34. Omuemu VO, Otasowie EM \& Onyiriuka U (2012) Prevalence of food insecurity in Egor local government area of Edo State, Nigeria. Ann Afr Med 11, 139-145.

35. Sanusi RA, Badejo CA \& Yusuf BO (2006) Measuring household food insecurity in selected local government areas of Lagos and Ibadan, Nigeria. Pak J Nutr 5, 62-67.

36. Belijo ZN \& Mensa M (2017) Levels and predictors of food insecurity among HIV positive adult patients taking highly active anti-retroviral therapy at Arba Minch general hospital, Southern Ethiopia, 2016. Gen Med (Los Angeles) 5, 30.

37. Kimani-Murage EW, Schofield L, Wekesah F et al. (2014) Vulnerability to food insecurity in urban slums: experiences from Nairobi, Kenya. J Urban Health 91, 1098-1113.

38. Perkins JM, Nyakato VN, Kakuhikire B et al. (2018) Food insecurity, social networks and symptoms of depression among men and women in rural Uganda: a cross-sectional, population-based study. Public Health Nutr 21 , 838-848.

39. Pérez-Escamilla R \& Segall-Corrêa A (2018) Food insecurity measurement and indicators. Rev Nutr 21, 15-26.

40. Oluma A, Abadiga M, Mosisa G et al. (2020) Food Insecurity among people living with HIV/AIDS on ART follower at public hospitals of Western Ethiopia. Int J Food Sci $\mathbf{2 0 2 0}$, 8825453.
41. Alaimo K, Olson CM \& Frongillo EA (2002) Family food insufficiency, but not low family income, is positively associated with dysthymia and suicide symptoms in adolescents. J Nutr 132, 719-725.

42. Tauer L (1995) Age and farmer productivity. Rev Agric Econ 17, 63-69.

43. Babatunde RO, Omotesho OA \& Sholotan OS (2007) Socioeconomics characteristics and food security status of farming households in Kwara state, North-Central Nigeria. PakJ Nutr 6, 49-58

44. Santos TGD, Silveira JACD, Longo-Silva G et al. (2018) Trends and factors associated with food insecurity in Brazil: the national household sample survey, 2004, 2009, and 2013]. Cad Saude Publica 34, e00066917.

45. Boneya DJ, Ahmed AA \& Yalew AW (2019) The effect of gender on food insecurity among HIV-infected people receiving anti-retroviral therapy: a systematic review and meta-analysis. PLoS One 14, e0209903. doi: 10.1371/journal.pone. 0209903.

46. Weiser SD, Frongillo EA, Ragland K, Hogg RS, Riley ED \& Bangsberg DR (2009) Food insecurity is associated with incomplete HIV RNA suppression among homeless and marginally housed HIV-infected individuals in San Francisco. J Gen Intern Med 24, 14-20.

47. Wang EA, McGinnis KA, Fiellin DA et al. (2011) Food insecurity is associated with poor virologic response among HIV-infected patients receiving antiretroviral medications. J Gen Intern Med 26, 1012-1018.

48. Normen L, Chan K, Braitstein P et al. (2005) Food insecurity and hunger are prevalent among HIV-positive individuals in British Columbia, Canada. J Nutr 135, 820-825.

49. Weiser SD, Bangsberg DR, Kegeles S, Ragland K, Kushel MB \& Frongillo EA (2009) Food insecurity among homeless and marginally housed individuals living with HIV/AIDS in San Francisco. AIDS Behav 13, 841-848.

50. Gregson S, Mushati P \& Nyamukapa C (2007) Adult mortality and erosion of household viability in AIDS-afflicted towns, estates, and villages in eastern Zimbabwe. J Acquir Immune Defic Syndr 44, 188-195.

51. Ngalula J, Urassa M, Mwaluko G et al. (2002) Health service use and household expenditure during terminal illness due to AIDS in rural Tanzania. Trop Med Int Health 7, 873-877.

52. Twine W \& Hunter LM (2011) Adult mortality and household food security in rural South Africa: does AIDS represent a unique mortality shock? Dev South Afr 28, 431-444.

53. Kushel MB, Gupta R, Gee L et al. (2006) Housing instability and food insecurity as barriers to health care among lowincome Americans. J Gen Intern Med 21, 71-77.

54. Vozoris NT \& Tarasuk VS (2003) Household food insufficiency is associated with poorer health. J Nutr 133, $120-126$. 